\title{
Self-quenching of the semiconductor laser linewidth below the Schawlow-Townes limit by using optical feedback
}

\author{
Y. Shevy, J. Iannelli, J. Kitching, and A. Yariv \\ Department of Applied Physics, M.S. 128-95, California Institute of Technology, Pasadena, California 91125
}

Received November 19, 1991

\begin{abstract}
We demonstrate theoretically and experimentally self-quenching of the fundamental semiconductor laser frequency fluctuations to a level that is orders of magnitude below the Schawlow-Townes limit for a solitary laser. It is shown that the main operative mechanism is the combined action of a frequency-dependent internal loss and amplitude-to-phase coupling. The internal frequency-dependent loss is introduced by means of spectrally narrow external optical feedback, which provides a strong frequency-dependent dispersion. Linewidth reduction by a factor of $2 \times 10^{3}$ is demonstrated by using a narrow Doppler-free Faraday resonance in Cs vapor.
\end{abstract}

The fundamental linewidth of semiconductor lasers is a topic of continuing interest. The quantum limit as derived by Schawlow and Townes varies as the square of the optical mode volume. In small-volume semiconductor lasers this translates typically to a linewidth of a few megahertz. In the case of semiconductors ${ }^{1-3}$ the linewidth is also increased by an additional factor $\left(1+\alpha^{2}\right)$, where $\alpha$, the amplitudeto-phase coupling factor, is in the range of 3-6.

In a previous Letter it was pointed out that the amplitude-to-phase coupling mechanism can be exploited to quench the semiconductor laser linewidth even below the Schawlow-Townes limit. ${ }^{4}$ This can be achieved by including a frequency-dependent loss mechanism in the laser cavity. In this Letter we show that a frequency-dependent optical feedback, produced by a narrow Doppler-free Faraday resonance from Cs vapor, can give rise to a few orders of magnitude reduction of the quantum-limited laser linewidth. We first review the basic predictions of our general model, then analyze the situation in which an external frequency-dependent element provides a weak optical feedback to the semiconductor laser. Finally we compare the predictions of the theory with our experimental results.

To model the effects of a frequency-dependent loss we use the Van der Pol laser oscillator driven by spontaneous emission. In Ref. 4 the dispersive loss mechanism was represented by a frequencydependent photon lifetime, $\tau_{p}{ }^{-1}=\tau_{p 0}{ }^{-1}+2 C \dot{\varphi}$. In this Letter we expand this model to include also the attendant frequency-dependent index change. This can be accounted for by allowing $C$ to be a complex quantity, $C=C_{r}+i C_{i}$, where $C_{r}$ represents a frequency-dependent loss and $C_{i}$ represents a frequency-dependent index change. The modified Van der Pol equations are therefore given by ${ }^{4}$

$$
\begin{aligned}
\dot{\delta}+\omega_{1} \delta+A_{0} C_{r} \dot{\varphi} & =\Delta_{i} / 2 \omega_{m}, \\
A_{0}\left(1+C_{i}\right) \dot{\varphi}-\alpha \omega_{1} \delta & =-\Delta_{r} / 2 \omega_{m} .
\end{aligned}
$$

With the use of Laplace transform methods the autocorrelation function for the laser phase is found to be

$$
\left\langle\varphi\left(t_{1}\right) \varphi\left(t_{2}\right)\right\rangle=\frac{W}{4 A_{0}^{2} \omega_{m}^{2}} \frac{\left(1+\alpha^{2}\right)}{\left(1+\alpha C_{r}+C_{i}\right)^{2}} \min \left(t_{1}, t_{2}\right) .
$$

Assumption of a white Langevin driving force to represent spontaneous emission leads to a Lorentzian field spectrum, $S_{E}(\omega)$, with the FWHM $\Delta \nu$ given by

$$
\Delta \nu=\Delta \nu_{\mathrm{S}-\mathrm{T}} \frac{\left(1+\alpha^{2}\right)}{\left(1+\alpha C_{r}+C_{i}\right)^{2}} \equiv \Delta \nu_{\mathrm{S}-\mathrm{T}} \frac{\left(1+\alpha^{2}\right)}{Q^{2}},
$$

where $\Delta \nu_{\mathrm{S}-\mathrm{T}}$ is the Schawlow-Townes limit.

In the case of a dominant $1 / f$ frequency fluctuation noise spectrum $\left[S_{\dot{\varphi}}(f) \sim 1 / f\right]$ it can be shown ${ }^{5}$ that the line shape of the laser field spectrum $S_{E}(\omega)$ becomes non-Lorentzian and can be approximated as a Lorentzian raised to the power $3 / 2$. Furthermore the laser linewidth is reduced only by $\Delta \nu=$ $\Delta \nu_{0} / Q$, where $\Delta \nu_{0}$ is the laser linewidth without feedback. Note that even in this case the frequency fluctuation spectrum $S_{\dot{\varphi}}(f)$ is reduced by a factor of $Q^{2}$. However, with $1 / f$ noise only the square root of the frequency fluctuations spectrum contributes to the width of the laser field, ${ }^{5} \Delta \nu \sim\left[S_{\dot{\varphi}}(1)\right]^{0.5} \sim 1 / Q$.

It is interesting to note the different roles played by $C_{r}$ and $C_{i}$ in the linewidth quenching process. A phase fluctuation of the laser leads to frequency change and consequently to amplitude change owing to $C_{r}$. This in turn leads, through the amplitude-tophase coupling mechanism, to a phase correction. Note that ironically the same amplitude-to-phase coupling mechanism normally gives rise to extra frequency noise owing to amplitude noise (the $1+\alpha^{2}$ term). On the other hand, $C_{i}$ yields directly a phase change since it represents the frequency-dependent refractive index. Hence only $C_{r}$ is multiplied by $\alpha$, which can make the contribution to the laser linewidth reduction that is due to $C_{r}$ larger than the $C_{i}$ mechanism for $\alpha>1$.

Aside from elucidating the different roles of the frequency-dependent amplitude and phase, Eq. (3) enables one to find the laser linewidth in various practical situations. In particular for our system, 


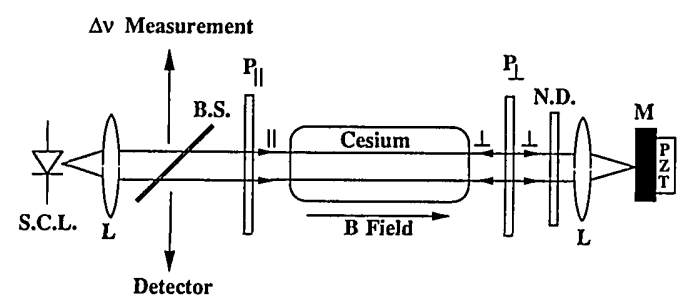

Fig. 1. Schematic layout of the experimental apparatus. S.C.L., semiconductor laser; L's, lenses; P's, linear polarizers; B.S., beam splitter; N.D., neutral-density filter; $\mathrm{M}$, mirror; PZT, piezoelectric transducer.

which is composed of a semiconductor laser with an external frequency-dependent reflector, we obtain

$$
\begin{aligned}
& C_{r}=\partial \kappa(\omega) / \partial \omega \cos \phi-\kappa(\omega) \sin \phi(\partial \phi / \partial \omega), \\
& C_{i}=\partial \kappa(\omega) / \partial \omega \sin \phi+\kappa(\omega) \cos \phi(\partial \phi / \partial \omega),
\end{aligned}
$$

where $\kappa(\omega)$ is the feedback coupling rate defined by $\kappa(\omega)=\left(1-r_{c}^{2}\right) r(\omega) / r_{c} \tau_{c}, r_{c}$ and $\tau_{c}$ are the semiconductor facet reflectivity and round-trip time, respectively, $r(\omega)$ is the frequency-dependent external reflectivity, $\phi$ is the feedback phase, $\partial \phi / \partial \omega=$ $\tau+\partial \phi^{\prime} / \partial \omega, \tau$ is the total external cavity round-trip time, and $\partial \phi^{\prime} / \partial \omega$ is the derivative of the optical feedback phase that is due to the dispersion of the external reflector. Using $C_{r}$ and $C_{i}$ we obtain for the linewidth reduction factor $Q$,

$$
\begin{aligned}
Q=1 & +\sqrt{1+\alpha^{2}}\left[\partial \kappa / \partial \omega \sin \left(\phi+\tan ^{-1} \alpha\right)\right. \\
& \left.+\kappa(\omega)\left(\tau+\partial \phi^{\prime} / \partial \omega\right) \cos \left(\phi+\tan ^{-1} \alpha\right)\right] .
\end{aligned}
$$

The operating frequency of the laser $\omega$ is given by the frequency pulling relation, ${ }^{6}$

$$
\omega=\Omega-\kappa(\omega) \sqrt{\left(1+\alpha^{2}\right)} \sin \left(\phi+\tan ^{-1} \alpha\right),
$$

where $\Omega$ is the laser frequency without feedback. We find experimentally that in the case of a narrow frequency-dependent feedback $\kappa(\omega)$, the laser tends to operate near zero frequency pulling. Thus only the cosine term in Eq. (4) is important. This means that the frequency-dependent dispersion is more important than the amplitude dependence of the feedback, $\partial \kappa / \partial \omega$. The dominant operating mechanism, however, is still the frequency-dependent amplitude change inside the semiconductor cavity. We conclude, therefore, that at zero frequency pulling the maximum linewidth reduction occurs at the peak of the frequency-dependent reflectivity. Assuming a Lorentzian $\kappa(\omega)$ with FWHM of $10 \mathrm{MHz}$ and peak amplitude of $2 \mathrm{GHz}$, we estimate $Q$ to be of the order of 1000. This should lead to a linewidth reduction in the case of a white frequency noise spectrum by a factor of $10^{6}$, a factor of $10^{6} /\left(1+\alpha^{2}\right) \sim 4 \times 10^{4}$ below the Schawlow-Townes limit for a solitary laser.

To test the theory, a frequency-dependent optical feedback was obtained by a Doppler-free Faraday effect in a Cs vapor. A 5-cm Cs cell was placed inside an external cavity composed by the laser's output facet and a mirror situated $40.5 \mathrm{~cm}$ away (see Fig. 1). The reflection from the external mirror was extinguished with two crossed linear polarizers (each with an extinction ratio of $1: 10^{4}$ ). The Cs cell was heated to $\sim 50^{\circ} \mathrm{C}$, and a magnetic field of $\sim 1 \mathrm{G}$ was applied along the laser's propagation axis. The laser was a Hitachi HLP 1400 and was operated single mode at $120 \mathrm{~mA}$ with a power of $12.5 \mathrm{~mW}$ and collimated to a spot size of $0.25 \mathrm{~cm}^{2}$.

This arrangement produced a narrow Dopplerfree reflection with a FWHM of $17 \mathrm{MHz}$ at the Cs $F=4 \rightarrow 5$ transition at $852 \mathrm{~nm}$ (similar reflection was obtained at the Cs $F=3 \rightarrow 2$ transition). The feedback phase and amplitude were controlled with a piezoelectric transducer at the back mirror and a variable neutral-density filter in front of it. The maximum feedback level was $-35 \mathrm{~dB}$ and was obtained at $\sim 1$ G. At higher magnetic fields the narrow emission was suppressed and stronger yet broader emissions with $\sim 200-\mathrm{MHz}$ FWHM became dominant. A 30\% beam splitter was used to monitor the feedback level and to direct part of the laser power to our measurement system. This system included a Newport Research supercavity with a resolution of $600 \mathrm{kHz}$ and a laser-linewidth-measuring apparatus that uses a delayed self-heterodyne technique with a fiber-optic delay line of $5 \mathrm{~km}$ to give a resolution of $6.4 \mathrm{kHz}$. In the following we describe experiments with the narrow $F=4 \rightarrow 5$ transition.

The application of the optical feedback gave rise to a dramatic quenching of the laser linewidth. We observed a maximum reduction by a factor of 2000 , from $20 \mathrm{MHz}$ down to $10 \mathrm{kHz}$ [see Fig. 2, curve (a)]. However, this is still well below the value expected from Eq. (4) that predicts linewidth reduction by a factor of $10^{6}$. This discrepancy could not be explained by the limited resolution of our system. Figure 2 shows another beat note signal having a linewidth larger than the resolution limit. To explain this discrepancy we fitted the signal to a Lorentzian line shape (the dashed curve) and to a Lorentzian raised to the power $3 / 2$ (the solid curve). As can be seen, the latter provides a much better fit. This signifies that the laser linewidth is dominated by $1 / f$ noise, and as a consequence the measured laser linewidth as a function of the feedback level should be represented by a $1 / Q$ dependence, as demonstrated in Fig. 3, curve (a).

In Fig. 3, curve (b), we also show the linewidth reduction that is due to the external cavity with the laser tuned off the $\mathrm{Cs}$ line and with the polarizers slightly uncrossed. As can be seen, the Cs reso-

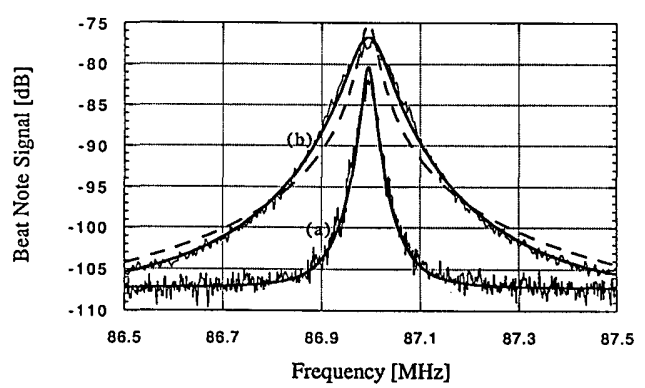

Fig. 2. Beat note obtained with delayed self-heterodyne. Curve (a) is the minimum linewidth obtained, with the solid curve being a best fit to a Lorentzian raised to the power $3 / 2$. Curve (b) is another beat note signal, with the dashed curve being a best fit to a Lorentzian line shape. 


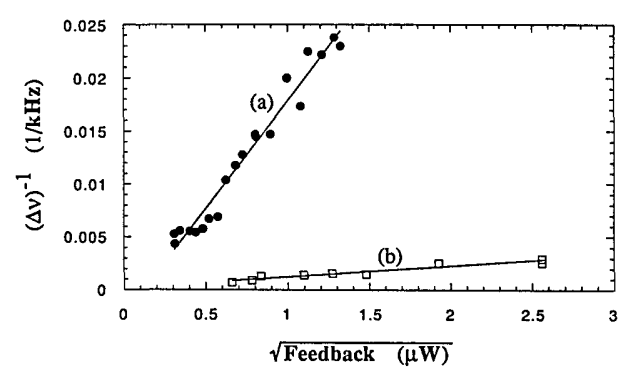

Fig. 3. $1 / \Delta \nu$ as a function of the square root of the feedback level with the linewidth measured at the top of the Faraday signal with zero frequency pulling [curve (a)] and an empty cavity [curve (b)].

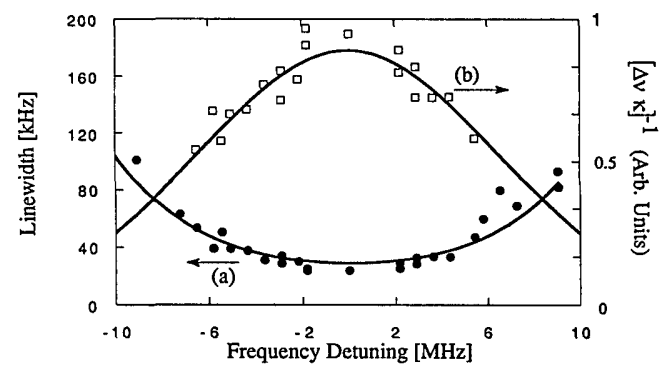

Fig. 4. Points on curve (a), the laser linewidth as a function of detuning from the peak of the Faraday signal. Points on curve (b), $[\Delta \nu \kappa(\omega)]^{-1}$ as a function of detuning; the solid curve is a fit to a derivative of dispersive line shape.

nance produces a much larger $Q$ than the empty cavity does. We note that the measurement of the linewidth in Fig. 3, curve (a), was done on the peak of the Cs transition, where only the frequencydependent dispersion of the $\mathrm{Cs}, \partial \phi^{\prime} / \partial \omega$, comes into play. Therefore the ratio between the slopes of the curves in Fig. 3 yields $\left(\partial \phi^{\prime} / \partial \omega+\tau\right) / \tau=16 \pm 1$.

In order to confirm independently the value of $\left(\partial \phi^{\prime} / \partial \omega+\tau\right)$, we performed a measurement of the frequency pulling as a function of the external mirror position (by modulating the piezoelectric voltage). Analysis of the frequency stability against feedback phase modulation by using Eq. (5) gives

$$
\frac{\partial \omega}{\partial \phi}=-\frac{\sqrt{\left(1+\alpha^{2}\right)} \kappa(\omega)}{1+\sqrt{\left(1+\alpha^{2}\right)}\left(\tau+\partial \phi^{\prime} / \partial \omega\right) \kappa(\omega)} .
$$

This measurement provided us with another means for evaluating $\left(\tau+\partial \phi^{\prime} / \partial \omega\right)$, which was found to be $(13 \pm 2) \tau$, in good agreement with the linewidth measurement $(16 \pm 1) \tau$.

In an additional effort to show that the frequencydependent dispersion that is due to the $\mathrm{Cs}$ resonance is the main contributor to $Q$, we measured the laser linewidth as a function of detuning from the peak of the Faraday signal while maintaining zero frequency pulling. Figure 4, curve (a), shows that the maximum reduction occurs at the peak of the Faraday signal. In order to demonstrate that this effect is not simply due to variation in $\kappa(\omega)$ but also due to variation in $\partial \phi^{\prime} / \partial \omega$, we plotted $[\Delta \nu \kappa(\omega)]^{-1}$ as a function of detuning [Fig. 4, curve (b)]. If the variations in linewidth were only due to $\kappa(\omega)$ a constant would be obtained, whereas curve (b) agrees with a derivative of a dispersive line shape derived from a
Lorentzian $\kappa(\omega)$. We note that a nonzero frequency pulling the frequency-dependent reflectivity also contributes. It was found experimentally that a small frequency pulling (obtained by varying the laser frequency with injection current slightly off the peak of the Faraday signal) gave rise to $30 \%$ improvement in laser linewidth reduction.

Finally we discuss this research in the context of other contributions in this field. In particular this research is related to the demonstration of frequency locking and linewidth reduction by optical feedback from a passive high-finesse confocal Fabry-Perot cavity. 7,8 In comparison, our system has the advantages of a long-term stability owing to a stable atomic transition frequency and simple alignment. On the other hand, the confocal Fabry-Perot technique offers the advantage of broadband tunability when needed. Lee and Campbell ${ }^{9}$ have demonstrated a similar Faraday technique in $\mathrm{Rb}$ vapor. Our research independently demonstrates the same effect in Cs vapor and in addition interprets and explains the results by an appropriate application of laser noise theory.

In conclusion, we have shown both theoretically and experimentally frequency noise reduction below the Schawlow-Townes limit for a solitary semiconductor laser. Our analysis indicates that the quantum limit of the linewidth as a result of spontaneous emission is reduced by a factor of $\sim 10^{6}$. However, owing to the important contribution of $1 / f$ noise, the reduction of the laser linewidth is only by a factor of 2000. We demonstrated that the operative mechanism in this dramatic effect is mainly the concerted action of frequency-dependent internal loss and amplitude-to-phase coupling, with an additional, smaller contribution that is due to internal frequency-dependent dispersion.

This research was supported by the Defense Advanced Research Projects Agency, the U.S. Office of Naval Research, and the U.S. Air Force Office of Scientific Research. We also thank M. Nakamura and T. Kajimura of Hitachi's Central Research Laboratories, Tokyo, Japan, for the generous donation of the laser used in our experiments.

\section{References}

1. F. Fleming and A. Mooradian, Appl. Phys. Lett. 8, 511 (1981).

2. C. H. Henry, IEEE J. Quantum Electron. QE-18, 259 (1982).

3. K. Vahala and A. Yariv, IEEE J. Quantum Electron. QE-19, 1096 (1983).

4. A. Yariv, R. Nabiev, and K. Vahala, Opt. Lett. 15, 1359 (1990).

5. D. Halford, presented at Frequency Standards and Metrology Seminar, Canada, 1971.

6. G. H. Agrawal, IEEE J. Quantum Electron. QE-20, 468 (1984).

7. B. Dahmani, L. Hollberg, and R. Drullinger, Opt. Lett. 12, 876 (1987).

8. P. Laurent, A. Clairon, and C. Breant, IEEE J. Quantum Electron. 25, 1131 (1989).

9. W. D. Lee and J. C. Campbell, Appl. Phys. Lett. 58, 995 (1991). 